\title{
Identification of candidate biomarkers for epithelial ovarian cancer metastasis using microarray data
}

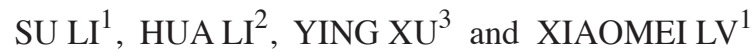 \\ ${ }^{1}$ Department of Obstetrics and Gynecology, Jinan Central Hospital Affiliated to Shandong University; ${ }^{2}$ Department of \\ Obstetrics and Gynecology, The People's Hospital of Zhangqiu, Jinan, Shandong 250014; ${ }^{3}$ Department of \\ Obstetrics and Gynecology, Shandong Coal Taishan Sanatorium, Taian, Shandong 271000, P.R. China
}

Received May 26, 2016; Accepted May 8, 2017

DOI: $10.3892 / \mathrm{ol} .2017 .6707$

\begin{abstract}
Epithelial ovarian cancer (EOC) is a common cancer in women worldwide. The present study assessed effective biomarkers for the prognosis of EOC metastasis. The GSE30587 dataset, containing 9 EOC primary tumor samples and 9 matched omental metastasis samples, was analyzed. Following normalization, the differentially expressed genes (DEGs) between these samples were identified using the limma package for R. Subsequently, pathway enrichment analysis was performed using ClueGO, and a protein-protein interaction (PPI) network was constructed using the Search Tool for the Retrieval of Interacting Genes database. The microRNA (mRNA/miR)-target network was established using the multiMiR package. A set of 272 DEGs was identified in metastatic EOC samples, including 189 upregulated and 83 downregulated genes. Collagen type I $\alpha 1$ chain (COL1A1), COL1A2, collagen type XI $\alpha 1$ chain (COL11A1) and thrombospondin $(T H B S) 1$ were enriched in the phosphoinositide 3-kinase/protein kinase B (PI3K/Akt), focal adhesion and extracellular matrix (ECM)-receptor interaction signaling pathways. THBSI and tissue inhibitor of metalloproteinase (TIMP) 3 were two dominant nodes in the PPI network and were key in the miRNA-target network, being targeted by hsa-miR-1. Multiple DEGs and miRNAs were identified as potential biomarkers for the prognosis of EOC metastasis in the present study, which likely affected metastasis by regulating the PI3K/Akt, ECM-receptor interaction and cell adhesion signaling pathways. In addition, THBSI and TIMP3 were identified as potential targets of hsa-miR-1.
\end{abstract}

Correspondence to: Professor Xiaomei Lv, Department of Obstetrics and Gynecology, Jinan Central Hospital Affiliated to Shandong University, 105 Jiefang Road, Zhangqiu, Jinan, Shandong 250014, P.R. China

E-mail: xiaomei582310@163.com

Key words: epithelial ovarian cancer, metastasis, microRNA, phosphoinositide 3-kinase/protein kinase B, cell adhesion

\section{Introduction}

Epithelial ovarian cancer (EOC) is the sixth largest cause of cancer-associated mortality in women globally (1). In 2012, $\sim 22,280$ and 69,565 cases of EOC were estimated for the USA and Europe, respectively (2). EOC accounts for $90 \%$ of cases of ovarian cancer and is characterized by metastasis (3). Typically, primary EOC tumors disseminate within the peritoneal cavity, primarily into the omentum (4). Only once the tumor cells have spread into the peritoneal cavity may EOC be diagnosed, which often results in a poor prognosis (5).

Numerous studies have assessed the mechanisms involved in EOC metastasis. Scotton et al (6) demonstrated that C-X-C motif chemokine receptor 4 was the only chemokine receptor expressed in ovarian cancer cells. This restricted expression is proposed to be a major step in ovarian cancer metastasis. Disrupting cell adhesion promotes tumor progression. The downregulation of the adhesion molecules cluster of differentiation (CD)82 and CD9 has been reported to be associated with the progression of ovarian cancer, particularly metastasis (7). Another study reported that the tumorigenicity-associated protein mucin 1 serves a function in EOC metastasis (8).

MicroRNAs (miRNAs/miRs) are small non-coding RNAs that serve key functions in the development of numerous types of cancer, including EOC, by regulating gene expression (9). A previous study examined the alteration of miRNAs during the development of EOC and, as expected, identified numerous differentially expressed miRNAs, including the overexpression of miR-200a, 200b, 200c and 141 (1). However, there are few reports of miRNAs associated with EOC metastasis.

A recent study identified differentially expressed genes (DEGs) between EOC primary tumors and metastases by microarray profiling (4). However, this previous study primarily concerned copy number variations (CNVs), which refers to variations caused by gene rearrangement, and the upregulation of the transforming growth factor $\beta$ signaling pathway. The results of this previous study suggested that although the clone (the altered genes corresponding to the CNVs) in metastasis and primary tumors was different, the tumor cells were adapting to the omental environment. Despite these results, the function of numerous other DEGs and their interactions in EOC remain unclear. Therefore, the present study re-analyzed the GSE30587 microarray dataset (4) to 
identify DEGs between primary tumor and omental metastatic tumor EOC cells. Furthermore, the present study performed term and pathway enrichment analyses, and protein-protein interaction (PPI) network construction. The present study also combined the DEG data with information on miRNAs in multiple databases to predict miRNA-target interactions. Through these comprehensive bioinformatical methods, the present study assessed effective biomarkers for the prognosis of EOC metastasis.

\section{Materials and methods}

Data resources. The GSE30587 microarray dataset (4) was downloaded from the Gene Expression Omnibus (GEO) database (www.ncbi.nlm.nih.gov/geo). Of the dataset, 9 primary tissue samples (control samples) and 9 matched omental metastatic tumor samples (metastatic samples) from patients with serous EOC were used in the present study. The platform used for the detection of this microarray data in the study by Brodsky et al (4) was the GeneChip ${ }^{\mathrm{TM}}$ Human Gene 1.0 ST Array (Affymetrix; Thermo Fisher Scientific, Inc., Waltham, MA, USA).

Pretreatment and differential analysis. Expression profiles from probe level and annotation profiles from the dataset were downloaded from the GEO database. Raw data in the expression profiles were preprocessed via robust multi-array average (RMA) normalization (10), allowing the expression values from probe level to correspond with those of the gene level, in accordance with the annotation profile. The average probe expression value was considered to be the gene expression value. The DEGs between control and metastatic samples were identified using the limma package (version 3.22.7) of $\mathrm{R}$ software (11). The cut-off values for DEG selection were a fold-change in expression of $\geq 1.5$ and $\mathrm{P}<0.05$.

Term and pathway enrichment analyses. The Cytoscape plugin ClueGO (11), which facilitates pathway enrichment analysis and classification of enriched terms, was used to perform the enrichment analysis. Information in the Kyoto Encyclopedia of Genes and Genomes (http://www.genome. $\mathrm{jp} / \mathrm{kegg} / \mathrm{pathway} . \mathrm{html}$ ) database was combined. Based on the results of ClueGO, a $\kappa$ coefficient that reflected the association between two pathways or two functional terms was calculated, with a threshold of 0.4. Similar functional terms were given the same color. The Pathview package (version 1.4.2) of R software (12), which reveals the location of DEGs in a pathway, was used to present the enriched pathway. $\mathrm{P}<0.05$ was considered to indicate a statistically significant pathway selection.

PPI network analysis of the DEGs. The Search Tool for the Retrieval of Interacting Genes (STRING) database (13) is a comprehensive database containing coexpression, co-occurrence, text-mining, fusion and protein interaction information. STRING uses a combined score (0-1) to assess reliability; the higher the score, the more reliable the interaction. In the present study, a combined score of 0.4 was used to establish the PPI network, which was visualized using Cytoscape. Each protein in the network served as a node, and the degree of a

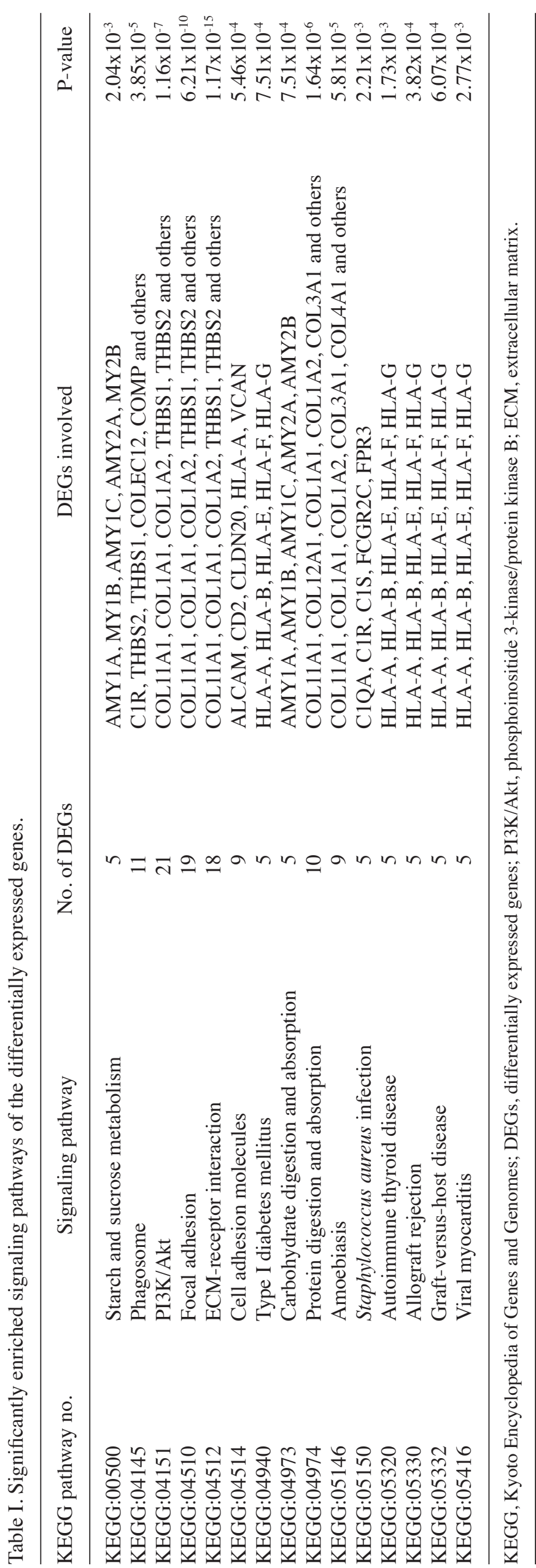



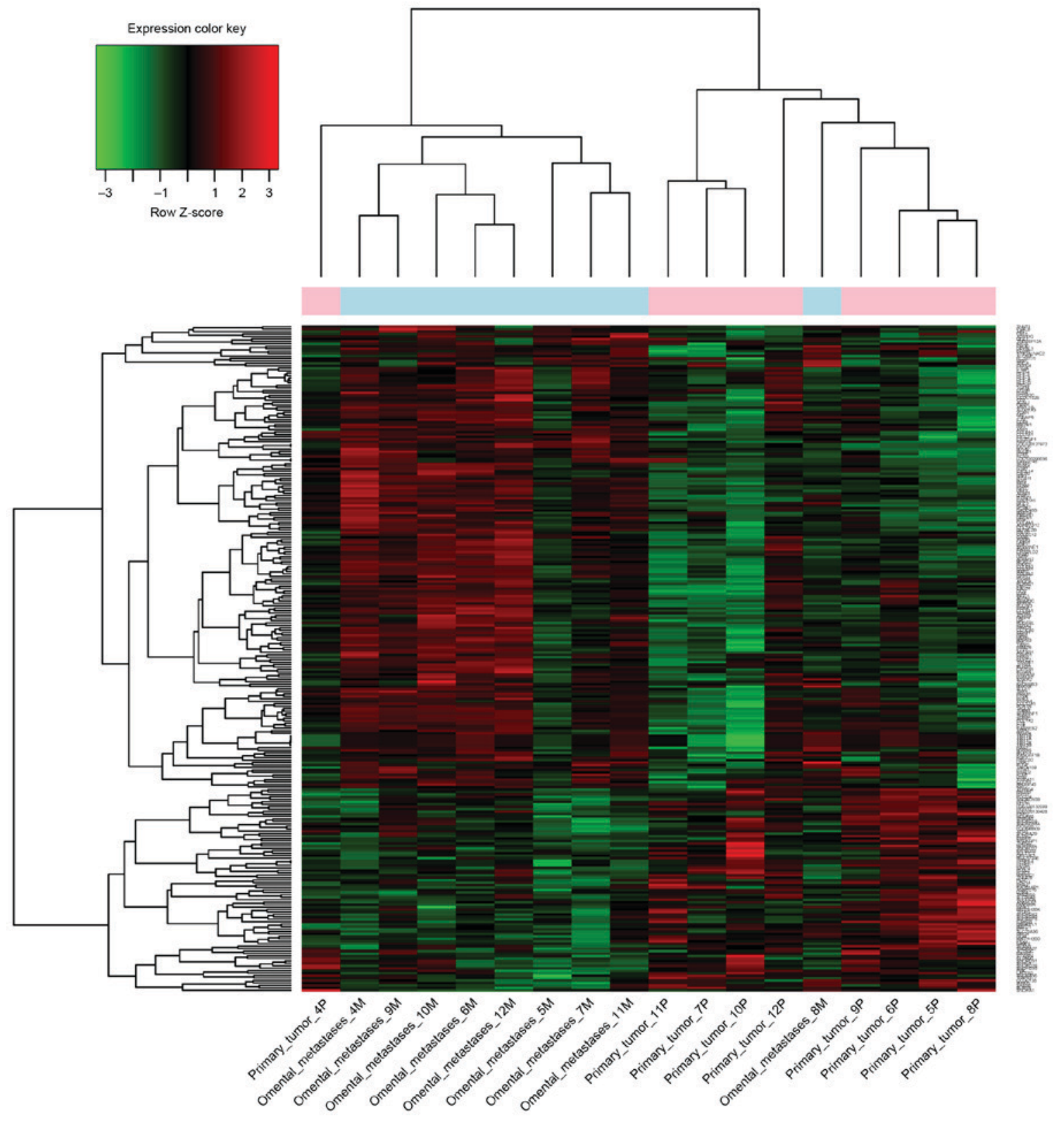

Figure 1. Heat map of gene expression in primary and metastatic epithelial ovarian cancer samples.

node was defined as the number of interactions with other nodes. Hub genes were nodes with $\geq 20$ degrees.

Construction of the miRNA-target regulatory network. The multiMiR package (version 3.0.2) (14) of R contains the miRNA-target interaction information from 14 databases, including three validated databases (miRecords version 4, miRTarBase version 4.5 and TarBase version 6), eight predicted databases (DIANA-microT-CDS version 5, E1MMo version 5, MicroCosm version 5, miRanda, miRDB version 4, PicTar version 2, PITA version 6 and TargetScan version 6.4) and three miRNA-disease/drug association databases [miR2Disease (version January, 2010), Pharmaco-miR (version 5.2) and PhenomiR (version 2.0)]. The present study extracted the miRNA-target interaction that appeared in at least two validated databases to establish the miRNA-target regulatory network. The network was subsequently visualized using Cytoscape.

\section{Results}

Identification of DEGs in metastatic EOC. The present study identified a total of 272 DEGs between the control and metastatic EOC samples, including 189 upregulated genes and 83 downregulated genes (Fig. 1).

Enriched signaling pathways of DEGs in metastatic EOS. With a predefined threshold of $\mathrm{P}<0.05$, the present study demonstrated that the DEGs identified in metastatic EOS were significantly enriched in signaling pathways associated with cellular signaling transduction and cell adhesion (Fig. 2; Table I), including the phosphoinositide 3-kinase/protein kinase B (PI3K/Akt) signaling pathway. This pathway included collagen type I a 1 chain (COL1A1), COL1A2, collagen type $\mathrm{XI} \alpha 1$ chain (COL11A1) and thrombospondin (THBS)1. The DEGs were also associated with the focal adhesion signaling 


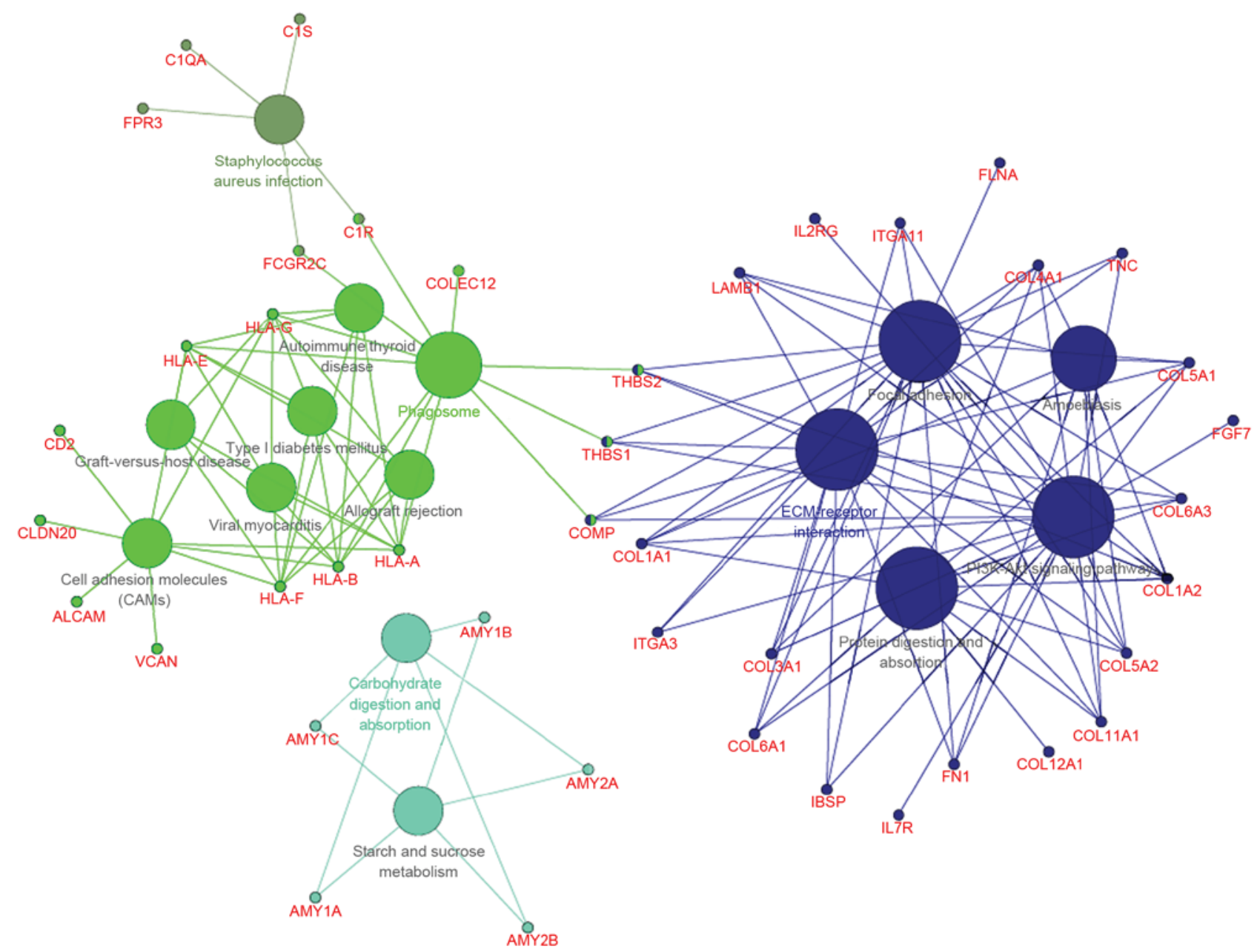

Figure 2. Enriched signaling pathways of the differentially expressed genes in metastatic EOC compared with primary EOC. EOC, epithelial ovarian cancer.

pathway, including COL1A1, COL1A2, COL11A1 and THBS1, the extracellular matrix (ECM)-receptor interaction signaling pathway, including COL1A1, COL1A2, COL11A1 and THBS1, and the cell adhesion signaling pathway, including activated leukocyte cell adhesion molecule and $C D 2$. DEGs enriched in the ECM-receptor interaction signaling pathway were all upregulated, including certain collagen genes and THBS (Fig. 3).

PPI network of DEGs in metastatic EOS. Using the STRING database, a PPI network consisting of 493 interactions of 146 DEGs was constructed (Fig. 4). The majority of the DEGs were upregulated, with the exception of 18 downregulated DEGs. A total of 14 hub genes were identified, including COL1AI (degree=37), matrix metallopeptidase $(M M P) 2($ degree $=36)$, decorin (degree=35), COL3A1 (degree=29), COL1A2 $($ degree=29), MMP14 $($ degree=26), COL5A1 (degree=26), secreted protein acidic and cysteine rich (degree=25), $C O L 4 A I$ (degree=25), THBS1 (degree=24), fibronectin 1 (degree=24), THBS2 (degree=22), tissue inhibitor of metalloproteinase $($ TIMP)3 (degree=21) and fibrillin 1 (degree=20).

Integrated miRNA-target gene regulatory network. The present study focused on the 14 hub genes, and assessed their miRNA-target associations further. The miRNA-target regulatory network was based on interactions in the aforementioned validated databases. THBS1 and TIMP3 were the dominant targets identified and interacted with multiple miRNAs (Fig. 5). THBS1 was predicted to be the target of the following eight miRNAs: hsa-miR-98-5p, hsa-let-7d-5p, hsa-miR-155-5p, hsa-let-7b-5p, hsa-miR-132-3p, hsa-miR-30a-3p, hsa-miR-30a-5p and hsa-miR-1. TIMP3 was predicted to be the target of seven miRNAs as follows: hsa-miR-124-3p, hsa-miR-21-5p, hsa-miR-181b-5p, hsa-miR-221-3p, hsa-miR-222-3p, hsa-miR-335-5p and hsa-miR-1.

\section{Discussion}

The present study identified several DEGs in metastatic EOS. Of these DEGs, certain collagen (COL11A1, COL1A1 and COL1A2) and THBS (THBS1 and THBS2) genes were associated with the PI3K/Akt, ECM-receptor interaction and cell adhesion signaling pathways. These DEGs were also hub genes in the PPI network constructed. THBS1 and TIMP3 dominated the miRNA-target network and were targeted by hsa-miR-1.

Hepatocyte growth factor (HGF) aids in the regulation of cell growth and motility. A previous study reported that HGF serves a crucial function in tumor metastasis by enhancing cell motility and increasing proteolytic activity in metalloproteases (15). The PI3K/Akt signaling pathway is a crucial kinase cascade involving HGF-induced metastasis and invasion (16). 


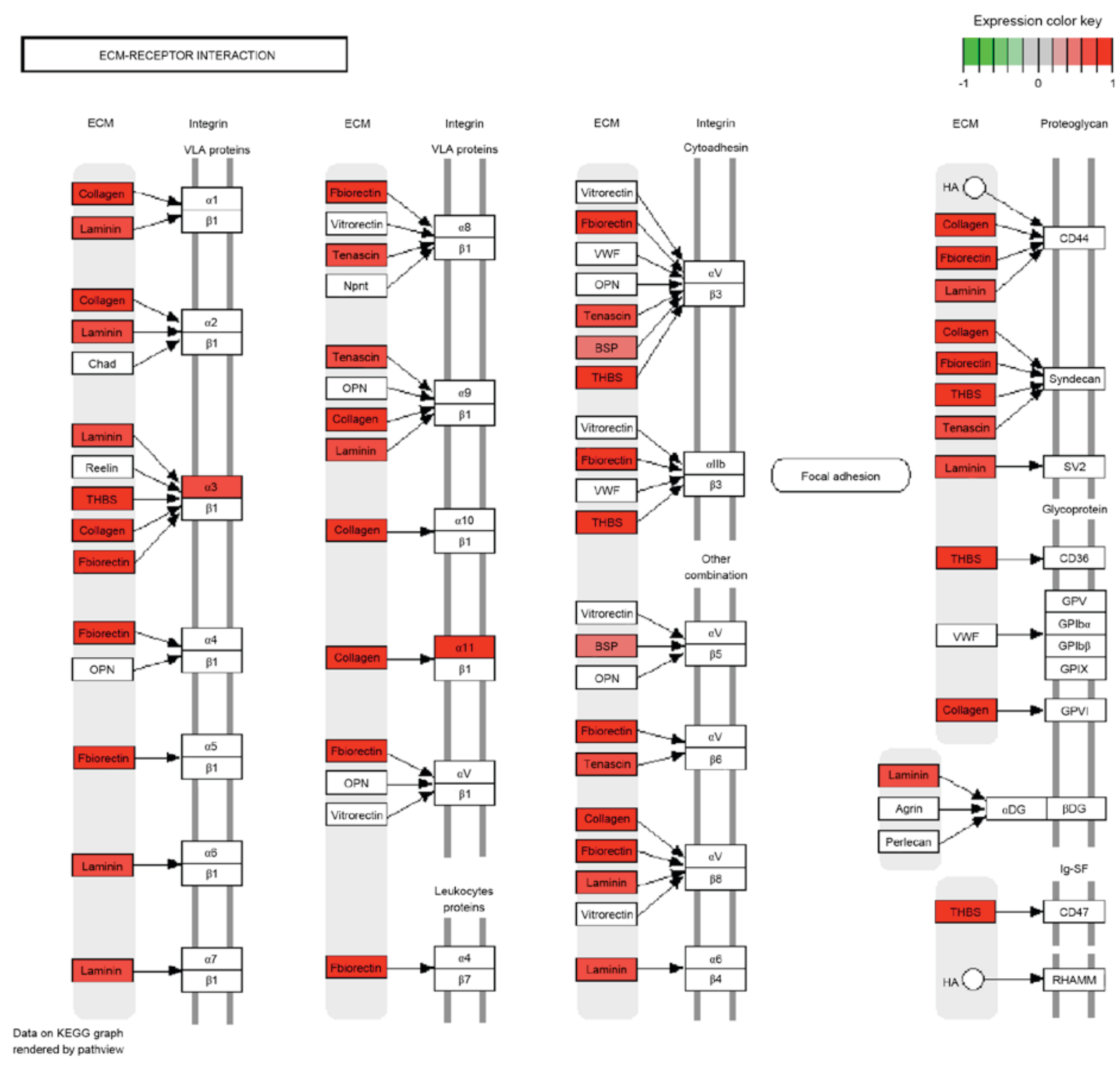

Figure 3. Differentially expressed genes enriched in the extracellular matrix-receptor interaction signaling pathway.

In uveal melanoma cells, activating the PI3K/Akt signaling pathway decreases cell adhesion, and thus promotes motility and migration (17). In glioma cells, the PI3K/Akt signaling pathway may regulate tumor cell proliferation and migration (18). Expression of collagen genes is often regulated via the PI3K/Akt signaling pathway. In hepatic stellate cells, collagen genes may be regulated by fascin, a component of actin bundles, through the focal adhesion kinase/PI3K/Akt signaling pathway (19). In normal human dermal fibroblasts, the transcription of collagen genes may be stimulated by interleukin-13 via the PI3K/Akt signaling pathway (20). In the present study, certain collagen genes, including $C O L 11 \mathrm{Al}$, COL1A1 and COL1A2, were identified as DEGs in metastatic EOC and were enriched in the PI3K/Akt signaling pathway, suggesting that these collagen genes may also serve functions in EOC through this signaling pathway, particularly in metastasis.

ECM proteolysis allows cancer cells to invade and is thus associated with migration in multiple types of cancer (21). A previous study demonstrated that COL11A1 promoted tumor progression in EOC via ECM-receptor interactions (22), a similar result to that which the present study revealed using enrichment analysis. In ovarian cancer cells, the ECM-receptor interaction signaling pathway is affected by COLIAl (23). COL1A2 is primarily associated with the cell adhesion signaling pathway in ovarian cancer cells (24). These results suggest that certain collagen genes, including COL11A1, COL1A1 and COL1A2, may also influence the metastasis of EOC through the ECM-receptor interaction and cell adhesion signaling pathways.

THBS1 is an adhesive glycoprotein that regulates cell-cell and cell-ECM interactions. A previous study demonstrated that THBS1 expression is associated with, and may function as a biomarker for the prognosis of, ovarian cancer (25). Another study demonstrated that downregulating THBS1 in ovarian cancer promotes tumor migration (26). According to comparative proteomic analysis, THBS1 is associated with cell adhesion, and differentially expressed between low malignant potential and highly proliferative EOC cell lines (27). THBS2 serves a function in cell-ECM adhesion (28). Furthermore, 


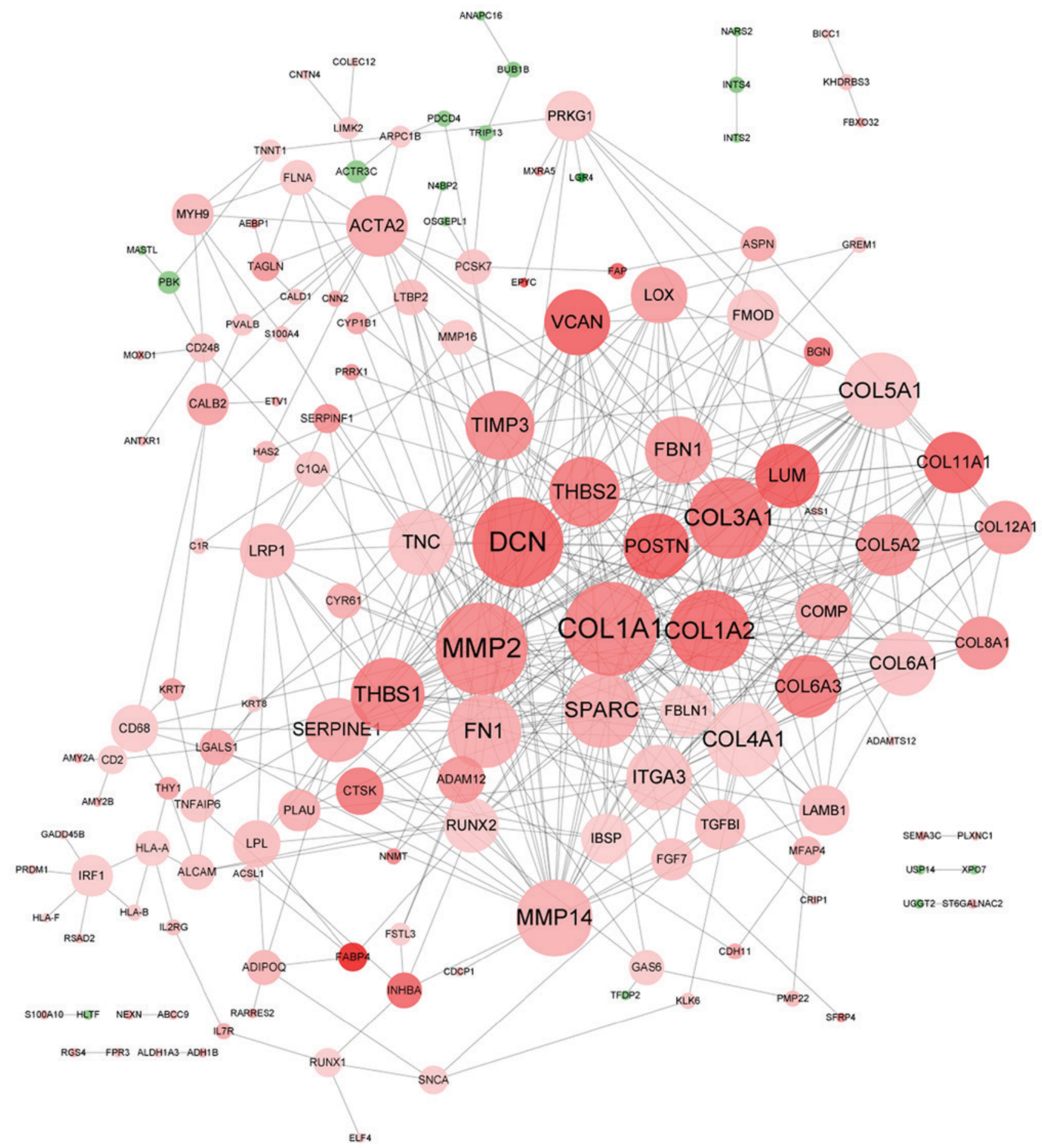

Figure 4. Protein-protein interaction network of the differentially expressed genes. Red nodes, upregulated genes; green nodes, downregulated genes.

THBS2 is one of ten signature genes associated with cell adhesion, and is associated with metastasis and poor overall survival time in patients with serous ovarian cancer (29). Downregulated by the inhibition of the Hedgehog signaling pathway, THBS1 is associated with ECM-ovarian cancer cell receptor interaction (30). The enrichment analysis performed in the present study demonstrated that THBS1 and THBS2 are associated with the cell adhesion and ECM-receptor interaction signaling pathways, suggesting they may serve key functions in EOC metastasis via regulating these two pathways.
TIMP3 inhibits MMPs, which are associated with ECM degradation. In osteosarcoma, lack of TIMP3 expression increases tumor cell proliferation and promotes migration (31). Arpino et al (32) demonstrated that TIMP3 serves a key function in the regulation of uterine ECM degradation during embryo implantation. Furthermore, TIMP3 was a key DEG identified in metastatic EOC in the present study. Although TIMP3 was not enriched in ECM-associated signaling pathways in the present study, TIMP3 was associated with THBS1 in the PPI network, suggesting that TIMP3 may serve a function in the ECM-receptor interaction signaling pathway during EOC metastasis. 


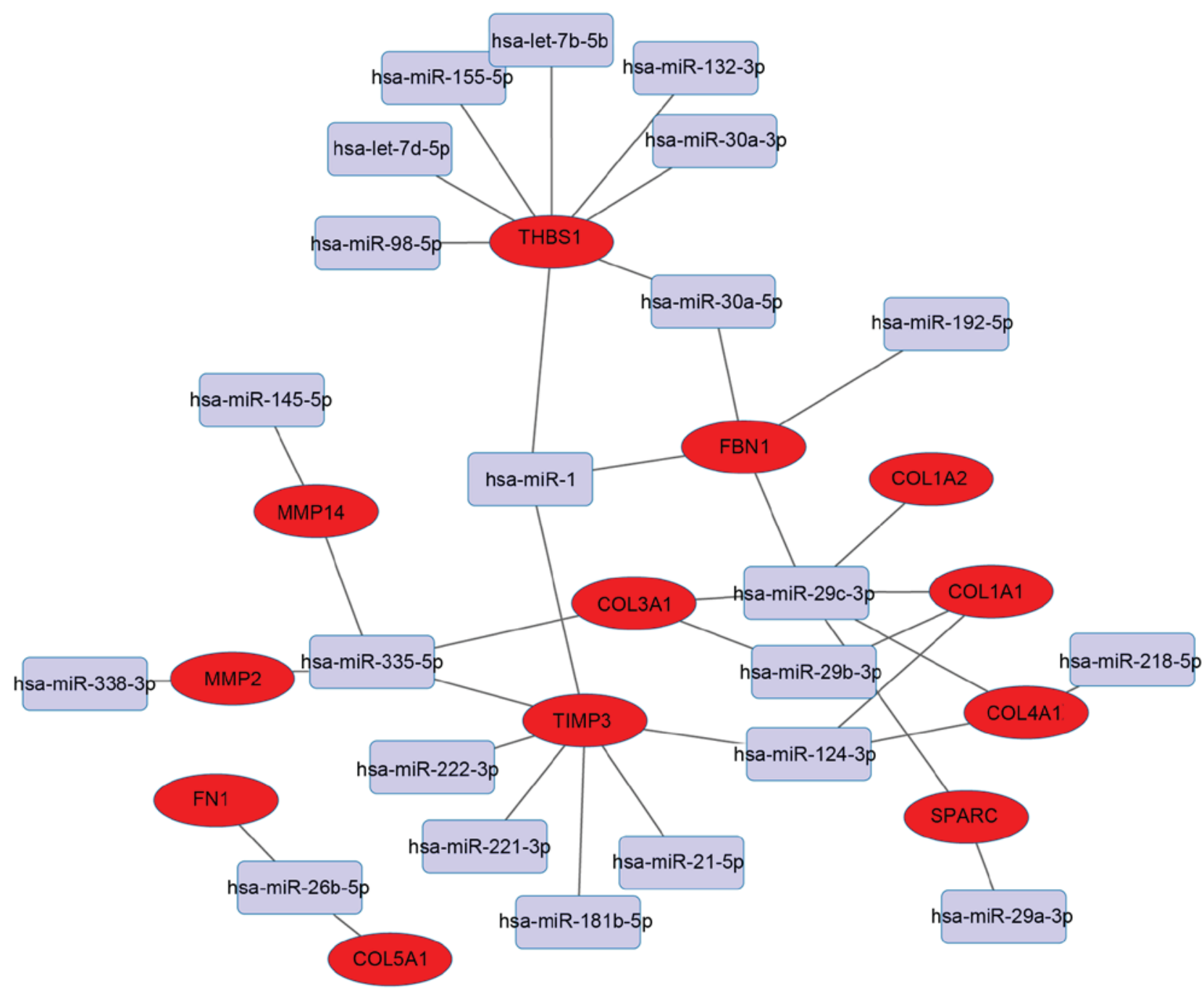

Figure 5. Integrated miRNA-target network. Red nodes, differentially expressed genes; lilac nodes, miRNAs.

Since hsa-miR-1 may decrease tumor cell proliferation in numerous types of cancer and is therefore considered a tumor suppressor. However, a previous study demonstrated that the upregulation of hsa-miR-1 was associated with increased tumor cell growth in relapsed ovarian tumors compared with ovarian primary tumors (33). In cardiac tissues, hsa-miR-1 may target TIMP3 (34) and is predicted to target THBS1 in heart failure (35). However, targeting of TIMP3 and THBSI by hsa-miR-1 has not yet been reported in EOC. In the present study, THBSI and TIMP3 were predicted as targets of hsa-miR-1, suggesting that hsa-miR-1 may target the two genes during EOC metastasis.

In conclusion, multiple DEGs and miRNAs were identified as potential biomarkers for the prognosis of EOC metastasis in the present study. These DEGs were associated with the PI3K/Akt, ECM-receptor interaction and cell adhesion signaling pathways. In addition, THBS1 and TIMP3 were predicted to be targets of hsa-miR-1. However, these predictive results require validation by further study.

\section{Acknowledgements}

The present study was supported by the Surface Project of Shandong Provincial Foundation (grant no.ZR2013HM097).

\section{References}

1. Iorio MV, Visone R, Di Leva G, Donati V, Petrocca F, Casalini P, Taccioli C, Volinia S, Liu CG, Alder H, et al: MicroRNA signatures in human ovarian cancer. Cancer Res 67: 8699-8707, 2007.

2. Aghajanian C, Blank SV, Goff BA, Judson PL, Teneriello MG, Husain A, Sovak MA, Yi J and Nycum LR: OCEANS: A randomized, double-blind, placebo-controlled phase III trial of chemotherapy with or without bevacizumab in patients with platinum-sensitive recurrent epithelial ovarian, primary peritoneal, or fallopian tube cancer. J Clin Oncol 30: 2039-2045, 2012.

3. Naora H and Montell DJ: Ovarian cancer metastasis: Integrating insights from disparate model organisms. Nat Rev Cancer 5: 355-366, 2005.

4. Brodsky AS, Fischer A, Miller DH, Vang S, MacLaughlan S, Wu HT, Yu J, Steinhoff M, Collins C, Smith PJ, et al: Expression profiling of primary and metastatic ovarian tumors reveals differences indicative of aggressive disease. PLoS One 9: e94476, 2014.

5. Lengyel E: Ovarian cancer development and metastasis. Am J Pathol 177: 1053-1064, 2010.

6. Scotton CJ, Wilson JL, Scott K, Stamp G, Wilbanks GD, Fricker S, Bridger G and Balkwill FR: Multiple actions of the chemokine CXCL12 on epithelial tumor cells in human ovarian cancer. Cancer Res 62: 5930-5938, 2002.

7. Houle CD, Ding XY, Foley JF, Afshari CA, Barrett JC and Davis BJ: Loss of expression and altered localization of KAI1 and CD9 protein are associated with epithelial ovarian cancer progression. Gynecol Oncol 86: 69-78, 2002.

8. Deng J, Wang L, Chen H, Li L, Ma Y, Ni J and Li Y: The role of tumour-associated MUC1 in epithelial ovarian cancer metastasis and progression. Cancer Metastasis Rev 32: 535-551, 2013. 
9. Zhang L, Volinia S, Bonome T, Calin GA, Greshock J, Yang N, Liu CG, Giannakakis A, Alexiou P, Hasegawa K, et al: Genomic and epigenetic alterations deregulate microRNA expression in human epithelial ovarian cancer. Proc Natl Acad Sci USA 105: 7004-7009, 2008.

10. Rich JN, Hans C, Jones B, Iversen ES, McLendon RE, Rasheed BK, Dobra A, Dressman HK, Bigner DD, Nevins JR and West M: Gene expression profiling and genetic markers in glioblastoma survival. Cancer Res 65: 4051-4058, 2005.

11. Bindea G, Mlecnik B, Hackl H, Charoentong P, Tosolini M, Kirilovsky A, Fridman WH, Pagès F, Trajanoski Z and Galon J: ClueGO: A Cytoscape plug-in to decipher functionally grouped gene ontology and pathway annotation networks. Bioinformatics 25: 1091-1093, 2009.

12. Luo W and Brouwer C: Pathview: An R/Bioconductor package for pathway-based data integration and visualization. Bioinformatics 29: 1830-1831, 2013.

13. Szklarczyk D, Franceschini A, Kuhn M, Simonovic M, Roth A Minguez P, Doerks T, Stark M, Muller J, Bork P, et al: The STRING database in 2011: Functional interaction networks of proteins, globally integrated and scored. Nucleic Acids Res 39 (Database issue): D561-D568, 2011.

14. Ru Y, Kechris KJ, Tabakoff B, Hoffman P, Radcliffe RA, Bowler R, Mahaffey S, Rossi S, Calin GA, Bemis L and Theodorescu D: The multiMiR R package and database: Integration of microRNA-target interactions along with their disease and drug associations. Nucleic Acids Res 42: e133, 2014.

15. Qian F, Engst S, Yamaguchi K, Yu P, Won KA, Mock L, Lou T, Tan J, Li C, Tam D, et al: Inhibition of tumor cell growth, invasion, and metastasis by EXEL-2880 (XL880, GSK1363089), a novel inhibitor of HGF and VEGF receptor tyrosine kinases. Cancer Res 69: 8009-8016, 2009.

16. Shi MD, Liao YC, Shih YW and Tsai LY: Nobiletin attenuates metastasis via both ERK and PI3K/Akt pathways in HGF-treated liver cancer HepG2 cells. Phytomedicine 20: 743-752, 2013.

17. Ye M, Hu D, Tu L, Zhou X, Lu F, Wen B, Wu W, Lin Y, Zhou Z and Qu J: Involvement of PI3K/Akt signaling pathway in hepatocyte growth factor-induced migration of uveal melanoma cells. Invest Ophthalmol Vis Sci 49: 497-504, 2008.

18. Chan KHB: Effect of CAL-101 on gene regulation of phosphoinositol 3-kinase isoform p110 $\delta$ in the pathogenesis of glioblastoma multiforme, 2013.

19. Uyama N, Iimuro Y, Kawada N, Reynaert H, Suzumura K, Hirano T, Kuroda N and Fujimoto J: Fascin, a novel marker of human hepatic stellate cells, may regulate their proliferation, migration, and collagen gene expression through the FAK-PI3K-Akt pathway. Lab Invest 92: 57-71, 2012.

20. Moriya C, Jinnin M, Yamane K, Maruo K, Muchemwa FC, Igata T, Makino T, Fukushima S and Ihn H: Expression of matrix metalloproteinase- 13 is controlled by IL- 13 via PI3K/Akt 3 and PKC- $\delta$ in normal human dermal fibroblasts. J Invest Dermatol 131: 655-661, 2011.

21. Wolf K and Friedl P: Mapping proteolytic cancer cell-extracellular matrix interfaces. Clin Exp Metastasis 26: 289-298, 2009.

22. Wu YH, Chang TH, Huang YF, Huang HD and Chou CY: COL11A1 promotes tumor progression and predicts poor clinical outcome in ovarian cancer. Oncogene 33: 3432-3440, 2014
23. Kumar S, Kumar A, Shah PP, Rai SN, Panguluri SK and Kakar SS: MicroRNA signature of cis-platin resistant vs. cis-platin sensitive ovarian cancer cell lines. J Ovarian Res 4: 17, 2011.

24. Cui J, Miner BM, Eldredge JB, Warrenfeltz SW, Dam P, Xu Y and Puett D: Regulation of gene expression in ovarian cancer cells by luteinizing hormone receptor expression and activation. BMC Cancer 11: 280, 2011

25. Abbott KL, Lim JM, Wells L, Benigno BB, McDonald JF and Pierce M: Identification of candidate biomarkers with cancer-specific glycosylation in the tissue and serum of endometrioid ovarian cancer patients by glycoproteomic analysis. Proteomics 10: 470-481, 2010.

26. Park K, Chung YJ, So H, Kim K, Park J, Oh M, Jo M, Choi K, Lee EJ, Choi YL, et al: AGR2, a mucinous ovarian cancer marker, promotes cell proliferation and migration. Exp Mol Med 43: 91-100, 2011.

27. Gagné JP, Éthier C, Gagné P, Mercier G, Bonicalzi ME, Mes-Masson AM, Droit A, Winstall E, Isabelle $M$ and Poirier GG: Comparative proteome analysis of human epithelial ovarian cancer. Proteome Sci 5: 16, 2007.

28. Ahmed AA, Mills AD, Ibrahim AE, Temple J, Blenkiron C, Vias M, Massie CE, Iyer NG, McGeoch A, Crawford R, et al: The extracellular matrix protein TGFBI induces microtubule stabilization and sensitizes ovarian cancers to paclitaxel. Cancer Cell 12: 514-527, 2007.

29. Cheon DJ, Tong Y, Sim MS, Dering J, Berel D, Cui X, Lester J, Beach JA, Tighiouart M, Walts AE, et al: A collagen-remodeling gene signature regulated by TGF- $\beta$ signaling is associated with metastasis and poor survival in serous ovarian cancer. Clin Cancer Res 20: 711-723, 2014.

30. Chen Q, Xu R, Zeng C, Lu Q, Huang D, Shi C, Zhang W, Deng L, Yan R, Rao H, et al: Down-regulation of Gli transcription factor leads to the inhibition of migration and invasion of ovarian cancer cells via integrin $\beta 4$-mediated FAK signaling. PLoS One 9: e88386, 2014

31. Han XG, Li Y, Mo HM, Li K, Lin D, Zhao CQ, Zhao J and Tang TT: TIMP3 regulates osteosarcoma cell migration, invasion, and chemotherapeutic resistances. Tumor Biol 37: 8857-8867, 2016

32. Arpino V, Brock M and Gill SE: The role of TIMPs in regulation of extracellular matrix proteolysis. Matrix Biol 44-46: 247-254, 2015.

33. Stope MB, Delogu S, Diesing K, Klinkmann G, Evert M, Koensgen D, Zygmunt M, Burchardt M and Mustea A: Expression pattern of the microRNA miR-1 in ovarian cancer cell lines and tumor tissue samples implies a loss of miR-1's tumor suppressor properties. Rna Disease 1: e384, 2014.

34. Vacchi-Suzzi C, Hahne F, Scheubel P, Marcellin M, Dubost V, Westphal M, Boeglen C, Büchmann-Møller S, Cheung MS, Cordier A, et al: Heart structure-specific transcriptomic atlas reveals conserved microRNA-mRNA interactions. PLoS One 8: e52442, 2013.

35. Tsoutsman T, Wang X, Garchow K, Riser B, Twigg S and Semsarian C: CCN2 plays a key role in extracellular matrix gene expression in severe hypertrophic cardiomyopathy and heart failure. J Mol Cell Cardiol 62: 164-178, 2013. 